\title{
Correspondence: Oncogenic MYC persistently upregulates the molecular clock component $\mathrm{REV}-\mathrm{ERB} \alpha$
}

\author{
Brian J. Altman ${ }^{1,2,3}$, Annie L. Hsieh ${ }^{1,2,3}$, Arvin M. Gouw ${ }^{1,2,3}$ \& Chi V. Dang1,2,3
}

Our report that MYC disrupts the circadian clock $^{1}$ was corroborated by Shostak et al. ${ }^{2}$ However, in contrast to our findings that MYC induces NR1D1 $(\mathrm{REV}-\mathrm{ERB} \alpha)^{1}$, Shostak et al. ${ }^{2}$ reported that overexpressed MYC in U2OS cells downregulated REV-ERB $\alpha$ and suppressed BMAL1 expression and the circadian clock through a MIZ1-dependent mechanism. They speculated that this discrepancy arose due to our documenting REV-ERB $\alpha$ expression at only a single-time point after MYC induction. Here, we present data in U2OS, other cancer cell lines, and a MYC-driven mouse model of liver cancer that MYC and N-MYC induce persistent upregulation of REV-ERB $\alpha$, which we thus believe to be a recurrent phenomenon downstream of oncogenic MYC. Furthermore, we note from both studies that REV-ERB $\alpha$ and MYC/MIZ1 may in fact cooperate in repressing BMAL1, pointing to a full consistency between the mechanisms revealed in either study.

Considering a number of experimental factors, we first authenticated our U2OS MYC-ER (oestrogen receptor Tamoxifen Mutant) BMAL1::Luc cells using a detailed 16 STR (short-terminal repeat) comparison ${ }^{3}$ (Supplementary Tables 1 and 2 and Supplementary Note 1). We next studied whether MYC induction of REV-ERB $\alpha$ in U2OS and other cell lines was timedependent. We treated U2OS MYC-ER with 4-hydroxytamoxifen (4OHT) or ethanol control for $24 \mathrm{~h}$, synchronized the cells with dexamethasone, and collected mRNA and protein every $4 \mathrm{~h}$. In two separate time-series experiments, we observed that MYC induction led to persistent upregulation of REV-ERB $\alpha$ (NR1D1) mRNA, upregulation of PER2 and downregulation of ARNTL (BMAL1) (Fig. 1a, Supplementary Fig. 1a). MYC also upregulated REV-ERB $\alpha$ protein (Fig. 1b, quantitated in Fig. 1c), which was elevated at nearly every time point assessed.

Given that we employed a MYC-ER system in U2OS cells whereas Shostak et al. ${ }^{2}$ utilized a MYC TET-ON model, we asked whether persistent REV-ERB $\alpha$ upregulation downstream of MYC was unique to MYC-ER, but we did not address the potential experimental artifacts associated with tetracycline ${ }^{4}$. We used two cell models with tetracycline-inducible MYC expression: mouse hepatocellular carcinoma cells derived from a tumour with conditional MYC TET-OFF expression ('mHCC 3-4'), and the MYC TET-OFF P493-6 human B cell line ${ }^{1,5-7}$. Notably, both mHCC 3-4 and p493-6 are normally cultured with elevated MYC protein which is then suppressed by tetracycline, and thus represent persistent models of MYC activation. In mHCC 3-4 cells, we observed that high MYC was associated with induced Nr1d1 (Rev-erb $\alpha$ ) and Per2 mRNA, while Arntl (Bmal1) was suppressed at later time points in high-MYC expressing cells (Supplementary Fig. 1b). Elevated MYC was also associated with increased protein expression of Rev-erb $\alpha$ and Cryl (which we previously showed was induced by MYC at the mRNA level ${ }^{1}$ ) and decreased Bmal1 (Supplementary Fig. 1c). P493-6 cells do not express detectable levels of BMAL1 mRNA, and thus have no discernible circadian oscillation. Nonetheless, after $2 \mathrm{~h}$ of serum shock $^{8}$, high-MYC expression in P493-6 cells was associated with persistently elevated REV-ERB $\alpha$ and PER2 expression (Supplementary Fig. 1d). P493-6 cells can also be induced to express intermediate levels of endogenous MYC protein with $\beta$-estradiol treatment ${ }^{9}$. In a time-series experiment conducted similarly to Supplementary Fig. 1d, we found that high MYC was associated with elevated REV-ERB $\alpha$ and PER2 (Supplementary Fig. 1e) as compared with intermediate MYC (Int. MYC) levels.

We previously demonstrated that N-MYC $(M Y C N)$, an oncoprotein functionally related to MYC and often elevated in neuroblastoma, bound at the NR1D1 promoter and transactivated REV-ERB $\alpha$ expression ${ }^{1}$. To examine whether N-MYC persistently induces REV-ERB $\alpha$ expression, we utilized two low MYCN-expressing neuroblastoma cell lines with inducible N-MYC, Shep N-MYC-ER ${ }^{10}$ and SKNAS N-MYC-ER ${ }^{11}$. These cells lines were treated with $4 \mathrm{OHT}$ or ethanol for $24 \mathrm{~h}$ to activate $\mathrm{N}-\mathrm{MYC}-\mathrm{ER}$, and then synchronized with dexamethasone. In both lines, including a replicate experiment with Shep N-MYC-ER, $\mathrm{N}-\mathrm{MYC}$ activation resulted in persistent upregulation of REV-ERB $\alpha$ and PER2 mRNA and suppression of BMAL1 mRNA (Fig. 1d, Supplementary Fig. 2a,b). Similarly, N-MYC

\footnotetext{
${ }^{1}$ Abramson Family Cancer Research Institute, Perelman School of Medicine, University of Pennsylvania, Philadelphia, Pennsylvania 19104, USA. ${ }^{2}$ Abramson Cancer Center, Perelman School of Medicine, University of Pennsylvania, Philadelphia, Pennsylvania 19104, USA. ${ }^{3}$ Division of Hematology-Oncology, Department of Medicine, Perelman School of Medicine, University of Pennsylvania, Philadelphia, Pennsylvania 19104, USA. Correspondence and requests for materials should be addressed to B.J.A. (email: altman@upenn.edu) or to C.V.D. 
a

U2OS MYC-ER
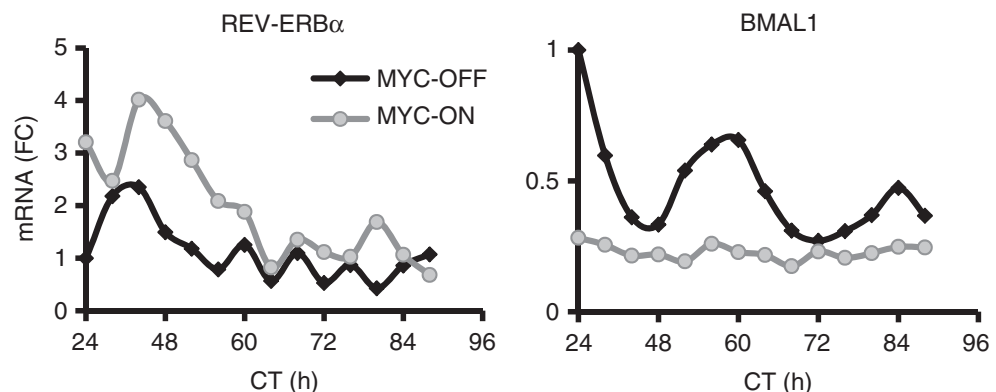

b

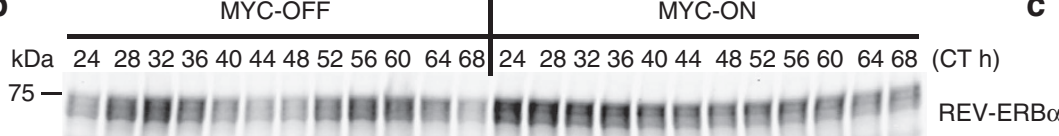

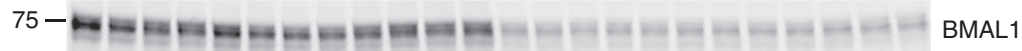

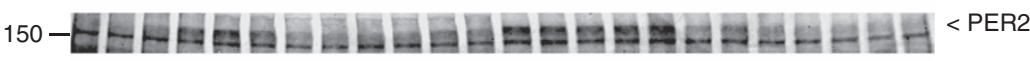
$50-$ -

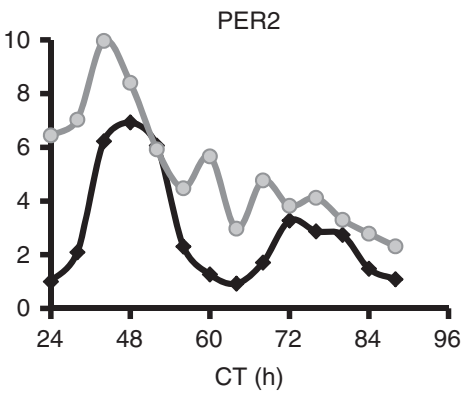

C

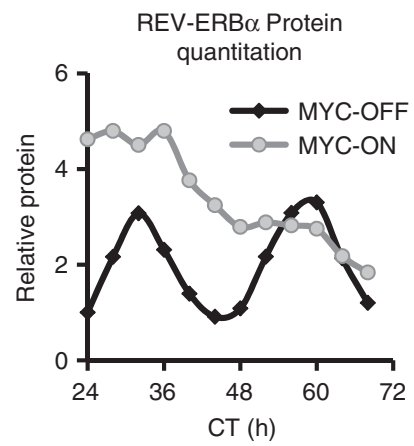

d

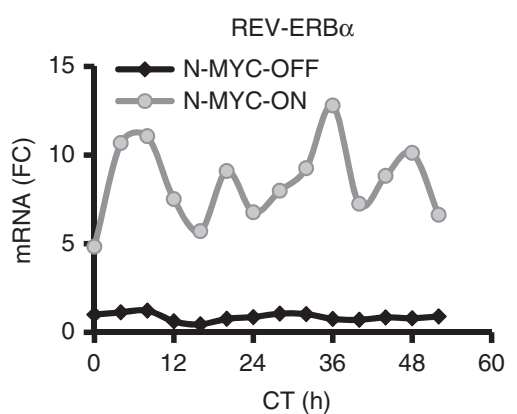

Shep N-MYC-ER

BMAL1

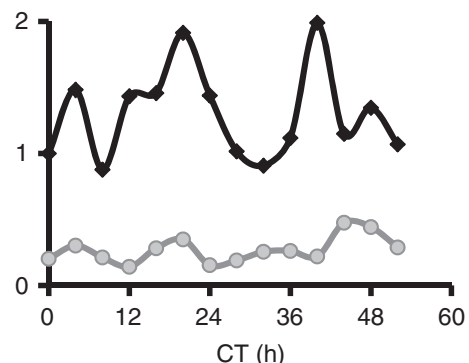

PER2

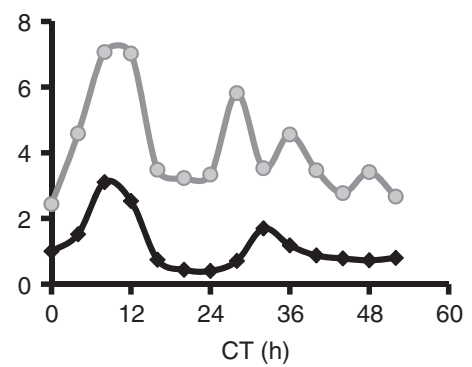

e

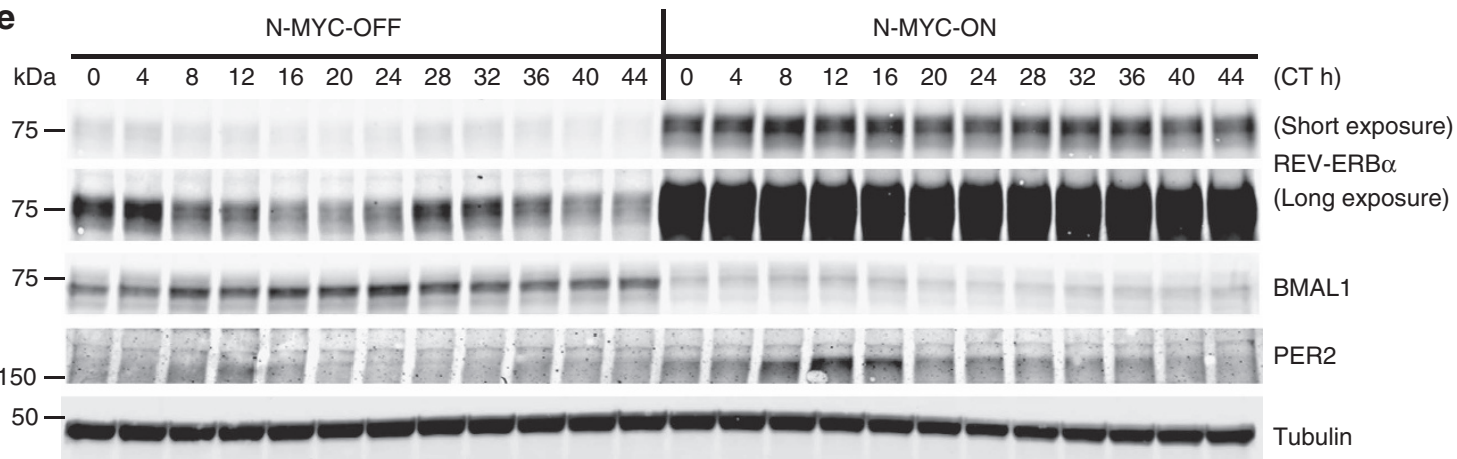

Figure 1 | MYC and N-MYC induce persistent elevation of REV-ERB $\boldsymbol{\alpha}$ mRNA and protein. (a,b) U2OS BMAL1::Luc cells expressing MYC-ER were cultured $\pm 4 \mathrm{OHT}$ (with ethanol serving as a loading control for all experiments) for $24 \mathrm{~h}$, then changed to 'lumicycle' media (see Methods) $\pm 4 \mathrm{OHT}$ and $+0.1 \mu \mathrm{M}$ dexamethasone. (a) mRNA was collected at the indicated time points after synchronization, and endogenous REV-ERB $\alpha$ (NR1D1), BMAL1 (ARNTL), and PER2 were determined by RT-PCR, normalized to $\beta 2 \mathrm{M}$. mRNA $(F C)=$ fold change. (b) Lysates were collected at the indicated time points after synchronization and processed for protein expression of REV-ERB $\alpha$, BMAL1, and PER2 (indicated by <). Tubulin serves as a loading control. Portions of $a$ and b were previously published ${ }^{1}$, but instead labelled as 0-48 h. (c) REV-ERB $\alpha$ protein from (b) was quantitated using Image Studio software (Licor, Lincoln, NE, USA), normalized to the relevant Tubulin and plotted over time. (d,e) SHEP N-MYC-ER expressing cells ${ }^{10}$ were cultured $\pm 40 H T$ for $24 \mathrm{~h}$, then $0.1 \mu \mathrm{M}$ dexamethasone was added. (d) mRNA was collected at the indicated time points after synchronization, and endogenous REV-ERB $\alpha$ (NR1D1), BMAL1 (ARNTL) and PER2, were determined by RT-PCR, normalized to $\beta 2 \mathrm{M}$. (e) Lysates were collected at the indicated time points after synchronization and processed for protein expression of REV-ERB $\alpha$ ('short' and 'long' indicate exposure time), BMAL1 and PER2. Tubulin serves as a loading control. For all panels, CT (cell time) indicate time of collection after synchronization. For immunoblots, molecular weights are noted in kDa. Portions of a-c were previously published ${ }^{1}$ and are reprinted with permission from Elsevier. 
a
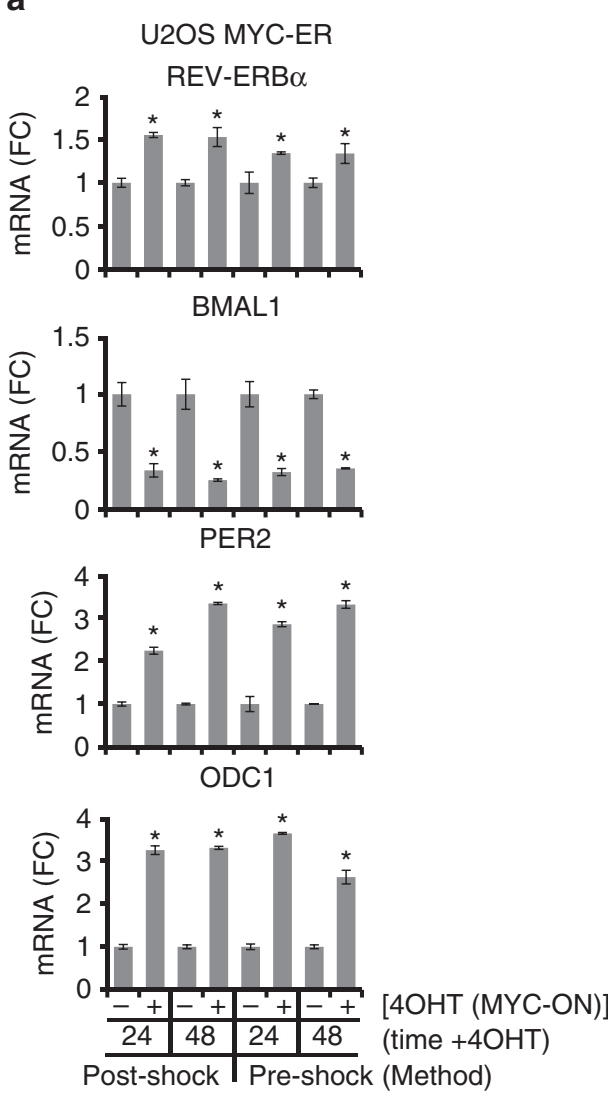

b

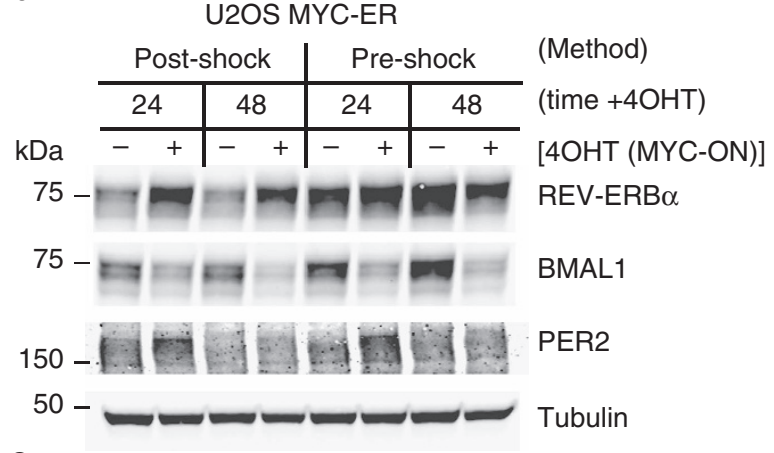

C

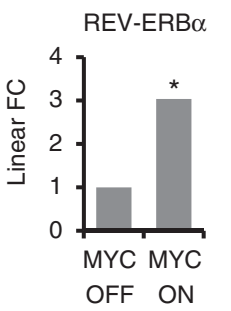

d

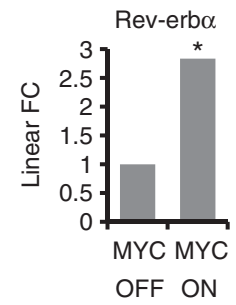

U2OS MYC TET-ON (30 h)

BMAL1

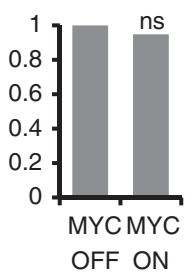

PER2

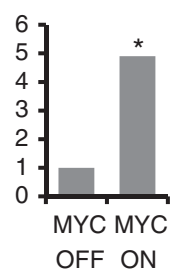

Mouse liver cancer MYC TET-OFF (16 h)

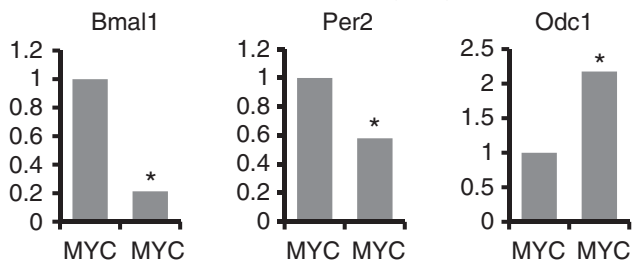

ODC1

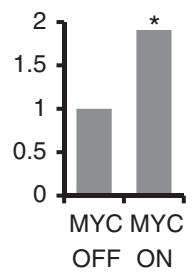

OFF ON

Figure 2 | REV-ERB $\alpha$ mRNA is induced by MYC regardless of synchronization method and in multiple cell line and tumor models. (a,b) MYC was induced in U2OS MYC-ER cells that were synchronized with dexamethasone in two different schemes. 'Post-Shock'1: as in Fig. 1, cells were treated \pm 4 OHT (with ethanol serving as a loading control for all experiments) for $24 \mathrm{~h}$. The ' 24 ' $\mathrm{h}$ sample was collected, then $0.1 \mu \mathrm{M}$ dexamethasone was added to media and the '48' $\mathrm{h}$ sample was collected $24 \mathrm{~h}$ later. 'Pre-Shock' 2 : $1 \mu \mathrm{M}$ dexamethasone was added for 20 min, then cells were washed once in PBS and fresh media was added $\pm 4 \mathrm{OHT}$. Cells were collected at the indicated times after media change. (a) mRNA was collected at the indicated times, and endogenous REV-ERB $\alpha$ (NR1D1), BMAL1 (ARNTL), PER2, and ODC1 were determined by RT-PCR, normalized to $\beta 2 \mathrm{M}$. mRNA (FC) = fold change. Data are averages of biological triplicates with error bars representing s.d., and ${ }^{\star} P<0.05$ by Student's $t$-test of 4 OHT (MYC-ON) samples relative to ethanol (MYC-OFF) samples at each time point. (b) Samples from (a) were processed for protein expression of REV-ERB $\alpha$, BMAL1 and PER2. Tubulin serves as a loading control. Molecular weights are noted in $\mathrm{kDa}$. (c) Previously published RNA-Seq data ${ }^{13}$ from U2OS cells expressing exogenous MYC under the control of a TET-ON system and treated $\pm 1 \mu \mathrm{g} \mathrm{ml} \mathrm{m}^{-1}$ doxycycline for $30 \mathrm{~h}$. REV-ERB $\alpha$ (NR1D1), BMAL1 (ARNTL), PER2 and ODC1 were determined. Data are presented as linear fold change (FC) and represent biological triplicates, and ${ }^{*} P<0.05$ of MYC-ON samples relative to MYC-OFF samples as previously described $^{13}$; NS, not significant. (d) Previously published RNA-seq data ${ }^{14}$ from liver tumors driven by a MYC-TET-OFF system. Mice were fed water or doxycycline for $16 \mathrm{~h}$ to turn off MYC. Rev-erb $\alpha$ (Nr1d1), Bmal1 (Arntl), Per2 and Odc1 were determined. Data are presented as linear FC and represent biological duplicates, and ${ }^{\star} P<0.05$ of MYC-ON samples relative to MYC-OFF samples as previously described ${ }^{14}$.

resulted in persistent upregulation of REV-ERB $\alpha$ protein and suppression of BMAL1 protein (Fig. 1e, Supplementary Fig. 2c). PER2 protein was also upregulated by N-MYC in both cell lines. Together, these results demonstrate in a total of five distinct cell lines that MYC or N-MYC activation results in persistent upregulation of REV-ERB $\alpha$.

We examined whether differences in clock synchronization and MYC induction methodology could affect REV-ERB $\alpha$ expression. We activated MYC for $24 \mathrm{~h}$ and then synchronized by stable addition of $0.1 \mu \mathrm{M}$ dexamethasone ('Post-Shock') ${ }^{1,12}$, while Shostak et al. ${ }^{2}$ transiently shocked cells for $20 \mathrm{~min}$ with $1 \mu \mathrm{M}$ dexamethasone ('Pre-Shock'), then washed and activated MYC after synchronization. We thus directly compared our PostShock with their Pre-Shock method and examined gene expression 24 and $48 \mathrm{~h}$ after MYC activation. With both methods, MYC significantly upregulated the mRNA expression of REV-ERB $\alpha, P E R 2$ and the canonical MYC target ODC1, while suppressing BMAL1, at both time points (Fig. 2a). We also examined protein expression, and found that with both methods MYC led to suppression of BMAL1 and early induction of PER2 (Fig. 2b). While the 'Post-Shock' method led to increased REV$\mathrm{ERB} \alpha$ protein at both 24 and $48 \mathrm{~h}$ after MYC induction, with the 'Pre-Shock' method we found REV-ERB $\alpha$ to already be highly elevated by dexamethasone compared to the unshocked sample ('Post-Shock' MYC-OFF 24h), and was not further elevated by MYC activation. Since MYC induced REV-ERB $\alpha$ mRNA with both methods, this non-MYC-stimulated increase in basal REV$\mathrm{ERB} \alpha$ protein may be due to increased protein production or stability. These results suggest that the different methods employed in synchronization and MYC activation both resulted in a significant induction of REV-ERB $\alpha$ mRNA by MYC over two time points.

We next analysed two previously published inducible MYC systems to determine whether MYC also induced REV- ERB $\alpha$ in 
these experiments. The Eilers laboratory employed a MYC-TETON U2OS model highly similar to Shostak et al., ${ }^{2}$ and published RNA-seq data $30 \mathrm{~h}$ after MYC induction ${ }^{13}$. In their data, MYC significantly induced REV-ERB $\alpha, P E R 2$ and ODC1 (Fig. 2c), though interestingly, BMAL1 was not suppressed at $30 \mathrm{~h}$. The Amati laboratory reported RNA-seq data for a MYC TET-OFF transgenic mouse model of liver cancer ${ }^{14}$. In these tumours, MYC expression was associated with elevated Rev-erb $\alpha$ and $O d c 1$ and suppressed Bmal1 (Fig. 2d), while Per 2 was suppressed by MYC, consistent with previous findings that MYC can suppress Per expression in some models ${ }^{15}$.

Collectively, the data suggest that MYC induces prolonged REV-ERB $\alpha$ expression in two MYC-inducible models of U2OS cells, four additional cell line models of inducible MYC and $\mathrm{N}-\mathrm{MYC}$, as well as primary transgenic inducible MYC liver cancer. However, the response of other clock genes, such as Per2, to MYC may be context-dependent ${ }^{15}$. It is also notable that in contrast to Shostak et al., ${ }^{2}$ who observed downregulation of $\mathrm{REV}-\mathrm{ERB} \alpha$ in the presence of ectopic MYC, we previously did not observe downregulation of REV-ERB $\alpha$ in control experiments with activation of MYC-ER $\Delta 106-143$ (ref. 1), which lacks full transactivation function but otherwise should retain the ability to interact with MIZ1. Nonetheless, double knockdown of both REV-ERB genes in our study partially rescued circadian oscillation of BMAL1::Luc in U2OS, suggesting that other mechanisms such as MIZ1 may also play a role in MYCdependent suppression of circadian oscillation (illustrated in Supplementary Fig. 3, a model of MYC disruption of circadian rhythm by multiple and likely overlapping mechanisms). In our comparative study reported here, we cannot explain the discrepancy between our data and those of Shostak et al. ${ }^{2}$ based on methodologic differences. The body of evidence we present here suggests that MYC induction of $\mathrm{REV}-\mathrm{ERB} \alpha$ is both persistent and recurrent across many inducible MYC model systems.

\section{Methods}

Cell culture. U2OS BMAL1::Luc MYC-ER (oestrogen receptor Tamoxifen Mutant) was derived by stable expression of pBabeZeo MYC-ER in U2OS BMAL1::Luc cells ${ }^{12}$, and subsequent selection and culture with $100 \mu \mathrm{g} \mathrm{ml}^{-1}$ Zeocin (Life Technologies, Grand Island, NY, USA) ${ }^{1}$. Murine hepatocellular carcinoma cell line (mHCC) 3-4, a primary culture tumour cell line, was derived by ring cloning of a liver tumour from the LAPtTA/tet-OFF cMYC conditional transgenic mouse liver cancer model $^{6,7}$. U2OS, mHCC 3-4, Shep N-MYC-ER ${ }^{10}$ and SKNAS N-MYC-ER ${ }^{11}$ were cultured in Dulbecco's Modified Eagle's Medium (DMEM, Mediatech, Manassas, VA, USA) containing glucose at $25 \mathrm{mM}$ and glutamine at $4 \mathrm{mM}$. Media was supplemented with $10 \%$ fetal bovine serum (FBS, HyClone, Logan, UT, USA or Life Technologies) and 1X Penicillin/ Streptomycin (Mediatech). mHCC cells were additionally supplemented with $2 \mathrm{mM}$ glutamine (Mediatech), $1 \mathrm{mM}$ sodium pyruvate (Mediatech) and 1X MEM non-essential amino acids (Life Technologies). P493-6 cells ${ }^{5,9}$ were cultured in RPMI-1640 medium (Mediatech) with $10 \%$ FBS (HyClone or Life Technologies) and 1X Penicillin/Streptomycin (Mediatech). All cell culture was conducted in a $5 \% \mathrm{CO}_{2}$ humidified atmosphere. For collecting cells, cells were washed one time in PBS (Life Technologies), removed from the plate with Trypsin-EDTA $0.25 \%$ (Life Technologies), suspended in media, spun down and then processed as indicated.

To activate MYC-ER or N-MYC-ER, U2OS, SHEP and SKNAS cells were treated with $500 \mathrm{nM}$ 4-hydroxytamoxifen (Sigma, St Louis, MO, USA) or ethanol control. To suppress MYC expression, mHCC 3-4 cells were treated with $20 \mathrm{ng} \mathrm{ml}^{-1}$ tetracycline (Sigma) or media control for 24 h, and P493-6 cells were treated with $100 \mathrm{ng} \mathrm{ml}^{-1}$ tetracycline or media control for $24 \mathrm{~h}$. To induce intermediate MYC expression (Int. MYC), P4936 cells were treated $+100 \mathrm{ng} \mathrm{ml}^{-1}$ tetracycline and $1 \mu \mathrm{M} \beta$ estradiol (Sigma) for 1 week, then cultured in $\beta$-estradiol \pm tetracycline for experiments.

U2OS BMAL1::Luc parental and MYC-ER were authenticated using the Cell Check 16 service (IDEXX Bioresearch, Columbia, MO, USA) with cell pellets that had been frozen. Authentication confirmed that the cells were free of interspecies contamination and genetically identical to a published U2OS profile using a detailed 16-STR (short tandem repeat) screen. U2OS BMAL1::Luc MYC-ER, mHCC 3-4 and p493-6 cells were tested for mycoplasma and found to be negative. SHEP N-MYC-ER and SKNAS N-MYC-ER were not tested.

Real-time PCR and primers. mRNA was extracted using the RNEasy Plus Mini Kit (Qiagen, Gaithersburg, MD, USA) following manufacturer's instruction and then reverse-transcribed to cDNA by using TaqMan Reverse Transcription Reagents (Life Technologies). cDNA was used as template for quantitative realtime PCR (RT-PCR) with specific human or mouse primers. All RT-PCRs in this work were performed using the ViiA 7 Real-time PCR system (Life Technologies). Relative mRNA expression levels were normalized to $\beta 2 M$ and analysed using comparative delta-delta CT method. RT-PCR primers and sources of these primers are listed in Supplementary Table 3, in the Supplementary Information section.

Circadian time-series sample collection. All circadian timeseries experiments were performed in the media described above, with U2OS, SHEP, SKNAS and mHCC 3-4 cells synchronized by addition of $0.1 \mu \mathrm{M}$ dexamethasone (Sigma) to growth media before start of collection. As an exception, for Fig. 1a-c, U2OS MYC-ER cells were switched to 'lumicycle media' containing dexamethasone at the start of the experiment: phenol red-free DMEM (Sigma) containing 5\% FBS (Hyclone or Life Technologies), $25 \mathrm{mM}$ D-glucose (Sigma), $35 \mathrm{mgl}^{-1}$ sodium bicarbonate (Thermo Fisher, Grand Island, NY), $10 \mathrm{mM}$ HEPES (Thermo Fisher) and Pen/Strep (Mediatech). P493-6 cells were 'serum shocked' in $50 \%$ FBS + growth media for $2 \mathrm{~h}$, then replated in normal growth media at the start of time-series collection ${ }^{8}$. All time-series experiments were performed as a 'split-timecourse': $24 \mathrm{~h}$ before to the start of the experiment, half the cells were synchronized with $0.1 \mu \mathrm{M}$ dexamethasone (dex, Sigma), and at the start of the experiment, the other half of the cells were synchronized with dexamethasone. At each time point, two plates were collected representing 0 and $24 \mathrm{~h}+\mathrm{dex}, 4$ and 28 and so on, to arrive at a $52 \mathrm{~h}$ time series. For all time-series experiments, CT denotes circadian time. mRNA and protein were processed and analysed as described above.

Comparison of synchronization methods. To compare synchronization methods between labs, we employed 'Pre-Shock'1,12: U2OS MYC-ER cells were first treated \pm 4 OHT for $24 \mathrm{~h}$, then some cells were collected for protein and mRNA $24 \mathrm{~h}$ later, and others were synchronized with $0.1 \mu \mathrm{M}$ dexamethasone and collected at $48 \mathrm{~h} \pm 4 \mathrm{OHT}$. For the method employed by Shostak et al. ${ }^{2}$ ('Post-Shock'), we treated U2OS MYC-ER cells with $1 \mu \mathrm{M}$ dexamethasone for $20 \mathrm{~min}$, washed once in PBS, then cultured the cells in normal medium \pm 4 OHT. Cells were collected for mRNA and protein 24 and $48 \mathrm{~h}$ later. 
Immunoblots and immunoblot quantitation. Cells were lysed for at least $20 \mathrm{~min}$ in M-PER Mammalian Protein Extraction Reagent (Thermo Fisher) supplemented with protease inhibitor cocktail (BD Biosciences, San Jose, CA, or Promega, Madison, WI, USA) and phosphatase inhibitors II and III (Sigma). Lysates were centrifuged at $16,000 \mathrm{~g}$ for $10 \mathrm{~min}$ at $4^{\circ} \mathrm{C}$, and supernatants were saved for quantitation and analysis. Protein content was quantified using the Bio-Rad DC assay kit (Bio-Rad, Hercules, CA, USA), with BSA serving as a reference (Thermo Fisher). Proteins were separated by SDS-PAGE using Criterion pre-cast gradient gels (Bio-Rad). Molecular weight was determined by comparison with Precision-Plus Dual Xtra protein standards (Bio-Rad). Primary antibodies used include: rabbit anti-REV-ERB $\alpha(1: 1,000, \# 13418 S$, Cell Signaling, Danvers, MA, USA); rabbit anti-BMAL1 (1:1,000, \#14020S, Cell Signaling); rabbit anti-PER2 (1:1,000, \#20359-1-AP, Proteintech, Chicago, IL, USA); rabbit anti-c-MYC (1:10,000, \#ab32072, Abcam, Cambridge, MA, USA); rabbit anti-CRY1 (1:1,000, \#ab104736, Abcam); and mouse anti- $\alpha$-Tubulin (1:10,000, \#CP06, EMD Millipore, Billerica, MA, USA). Secondary antibodies used include: Alexa-Flour 680 goat anti-rabbit IgG (1:8,000, \#A21109, Life Technologies, Grand Island, NY); Alexa-Flour 790 goat anti-mouse IgG (1:8,000, \#A11357, Life Technologies); and IRDye 800CW Goat Anti-Mouse IgG (1:10,000, \#926-32210, Licor, Lincoln, NE). Immunoblots were imaged with the Odyssey CLx infrared imaging system (Licor) and uniformly contrasted.

Immunoblots were quantitated using the Licor Image Studio software. For each band, median background intensity with a 3 pixel border width was automatically subtracted. Each REV$\mathrm{ERB} \alpha$ band value was normalized against the applicable Tubulin band, and then all the quantitated bands were further normalized by arbitrarily setting the MYC-OFF CT 24 value to 1 and dividing all the others by this value.

Raw, uncropped images of all immunoblots are presented in Supplementary Figs 4-8, along with molecular weight markers for each blot.

Statistical analysis. For all single-time point mRNA expression data with biological replicates, error bars represent s.d., and ${ }^{\star} P<0.05$ by Student's $t$-test from three experiments. For publically available data, ${ }^{*} P<0.05$ as previously described ${ }^{13,14}$.

Data availability. For U2OS MYC TET-ON RNA-Seq, publically available data was used from Walz et al. ${ }^{13}$ (GEO accession \# GSE44672, sample ID \# GSM1231609). Data were supplied as log fold-change and were linearized for this work. For mouse hepatocellular carcinoma MYC TET-OFF samples, publically available data was used from Kress et al. ${ }^{14}$, supplied in a Supplementary Figure to their work. Data were supplied as log fold-change and were linearized for this work. All other relevant data are available from the authors.

\section{References}

1. Altman, B. J. et al. MYC disrupts the circadian clock and metabolism in cancer cells. Cell Metab. 22, 1009-1019 (2015)

2. Shostak, A. et al. MYC/MIZ1-dependent gene repression inversely coordinates the circadian clock with cell cycle and proliferation. Nat. Commun. 7, 11807 (2016).

3. Yu, M. et al. A resource for cell line authentication, annotation and quality control. Nature 520, 307-311 (2015).
4. Moullan, N. et al. Tetracyclines disturb mitochondrial function across eukaryotic models: a call for caution in biomedical research. Cell Rep. 10, 1681-1691 (2015).

5. Schuhmacher, M. et al. Control of cell growth by c-Myc in the absence of cell division. Curr. Biol. 9, 1255-1258 (1999).

6. Shachaf, C. M. et al. MYC inactivation uncovers pluripotent differentiation and tumour dormancy in hepatocellular cancer. Nature 431, 1112-1117 (2004).

7. Xiang, Y. et al. Targeted inhibition of tumor-specific glutaminase diminishes cell-autonomous tumorigenesis. J. Clin. Invest. 125, 2293-2306 (2015).

8. Balsalobre, A., Damiola, F. \& Schibler, U. A serum shock induces circadian gene expression in mammalian tissue culture cells. Cell 93, 929-937 (1998)

9. Yustein, J. T. et al. Induction of ectopic Myc target gene JAG2 augments hypoxic growth and tumorigenesis in a human B-cell model. Proc. Natl Acad. Sci. USA 107, 3534-3539 (2010).

10. Ushmorov, A. et al. N-myc augments death and attenuates protective effects of Bcl-2 in trophically stressed neuroblastoma cells. Oncogene 27, 3424-3434 (2008).

11. Valentijn, L. J. et al. Inhibition of a new differentiation pathway in neuroblastoma by copy number defects of $\mathrm{N}-\mathrm{myc}, \mathrm{Cdc} 42$, and $\mathrm{nm} 23$ genes. Cancer Res. 65, 3136-3145 (2005).

12. Baggs, J. E. et al. Network features of the mammalian circadian clock. PLoS Biol. 7, e52 (2009).

13. Walz, S. et al. Activation and repression by oncogenic MYC shape tumourspecific gene expression profiles. Nature 511, 483-487 (2014).

14. Kress, T. R. et al. Identification of MYC-dependent transcriptional programs in oncogene-addicted liver tumors. Cancer Res. 76, 3463-3472 (2016).

15. Repouskou, A. \& Prombona, A. c-MYC targets the central oscillator gene Per1 and is regulated by the circadian clock at the post-transcriptional level. Biochim. Biophys. Acta 1859, 541-552 (2016).

\section{Acknowledgements}

This work is partially supported by the National Cancer Institute (NCI) of the National Institutes of Health (NIH) K99CA204593 (B.J.A.), 1T32CA196585-1 (A.M.G.), R01CA057341 (C.V.D.), The Leukemia and Lymphoma Society LLS 6106-14 (C.V.D.) and the Abramson Family Cancer Research Institute.

\section{Author contributions}

Conceptualization: B.J.A., C.V.D.; methodology: B.J.A., A.L.H., A.M.G, C.V.D.; validation: B.J.A., A.L.H., A.M.G.; formal analysis: B.J.A.; investigation: B.J.A., A.L.H., A.M.G.; resources: C.V.D.; writing-original draft: B.J.A., C.V.D.; writing-review and editing: B.J.A., A.L.H., A.M.G., C.V.D.; visualization: B.J.A., A.L.H.; supervision: C.V.D.; funding acquisition: B.J.A., C.V.D.

\section{Additional information}

Supplementary Information accompanies this paper at http://www.nature.com/ naturecommunications

Competing interests: The authors declare no competing financial interests.

Reprints and permission information is available online at http://npg.nature.com/ reprintsandpermissions/

How to cite this article: Altman, B. J. et al. Correspondence: Oncogenic MYC persistently upregulates the molecular clock component REV-ERB $\alpha$. Nat. Commun. 8, 14862 doi: 10.1038/ncomms14862 (2017).

Publisher's note: Springer Nature remains neutral with regard to jurisdictional claims in published maps and institutional affiliations.

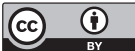

This work is licensed under a Creative Commons Attribution 4.0 International License. The images or other third party material in this article are included in the article's Creative Commons license, unless indicated otherwise in the credit line; if the material is not included under the Creative Commons license, users will need to obtain permission from the license holder to reproduce the material. To view a copy of this license, visit http://creativecommons.org/licenses/by/4.0/

(C) The Author(s) 2017 